\title{
Ultrasound for Critical Care Physicians: Ghost in the Machine
}

\author{
Ross Davidson, DO \\ Michel Boivin, MD \\ Division of Pulmonary, Critical Care and Sleep Medicine \\ University of New Mexico School of Medicine \\ Albuquerque, NM USA
}

A 53-year-old woman presented to the emergency department after a sudden cardiac arrest at home. The patient had a history of asthma and tracheal stenosis and had progressive shortness of breath over the previous days. The patient's family noticed a "thump" sound from the patient's room, and found her apneic. They called 911 and began cardiopulmonary resuscitation. Paramedics arrived on the scene, found an initial rhythm of pulseless electrical activity. The patient eventually achieved return of spontaneous circulation and was transported to the hospital. On arrival the patient was in normal sinus rhythm, with a heart rate of 110 beats per minute. Blood pressure was $80 / 45 \mathrm{mmHg}$, on an epinephrine infusion. The patient was afebrile, endotracheally intubated, unresponsive and ventilated at 30 breaths per minute. An initial chest radiograph was compatible with aspiration pneumonitis and a small pneumothorax. Initial electrocardiogram on arrival had $1 \mathrm{~mm}$ ST-segment depressions in leads V4 to V6. Transthoracic echocardiography was unsuccessful due to patient's habitus and mechanical ventilation. Because of the patient's hemodynamic instability and unknown cause of cardiac arrest, an urgent trans-esophageal echocardiogram (TEE) was performed (Videos 1-3).

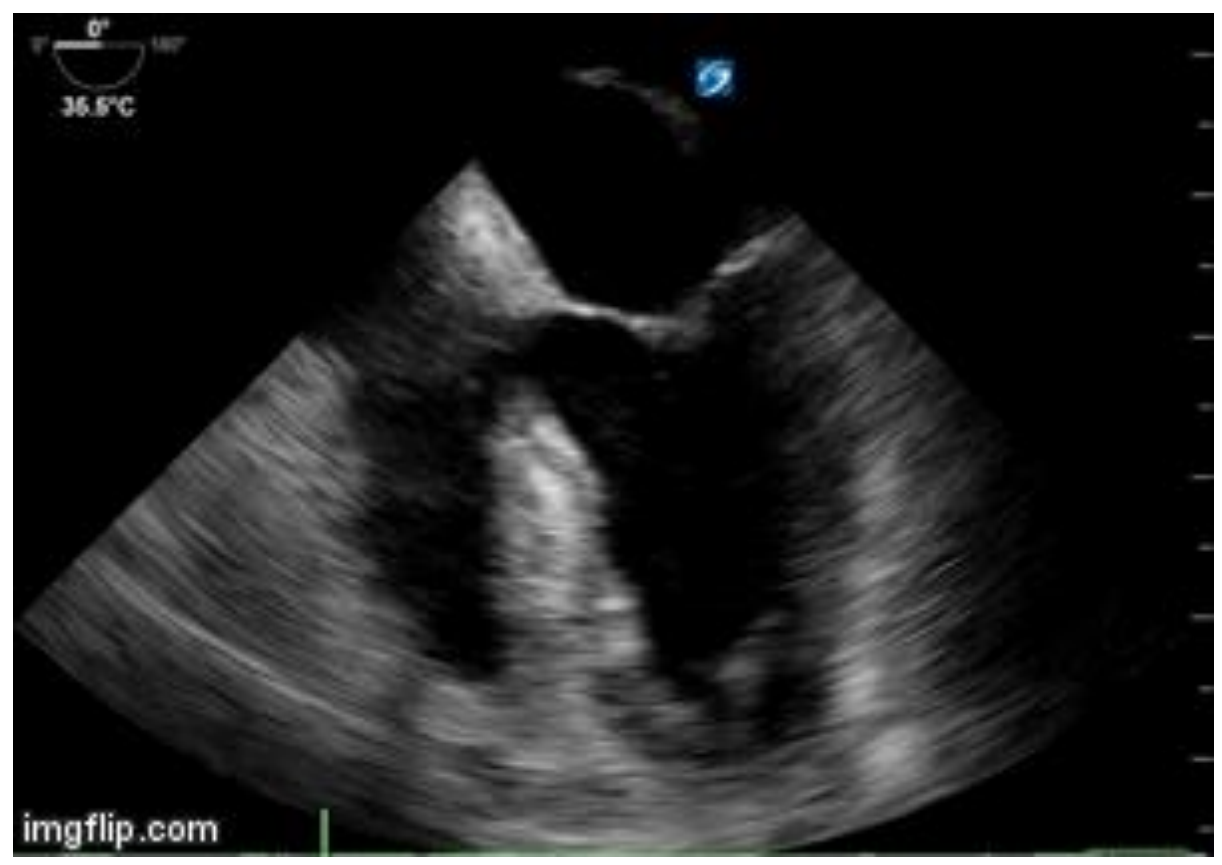

Video 1. Mid-esophageal 4-chamber view of the heart. 


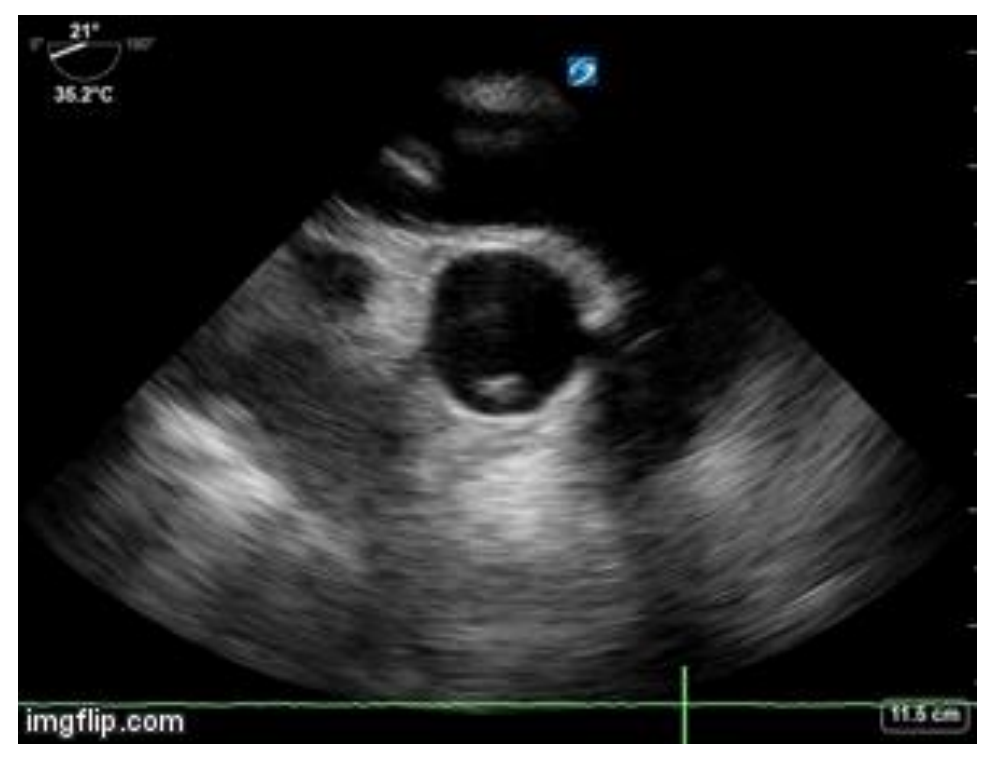

Video 2. Upper esophageal long-axis view of the pulmonary artery and short axis view of the ascending aorta.

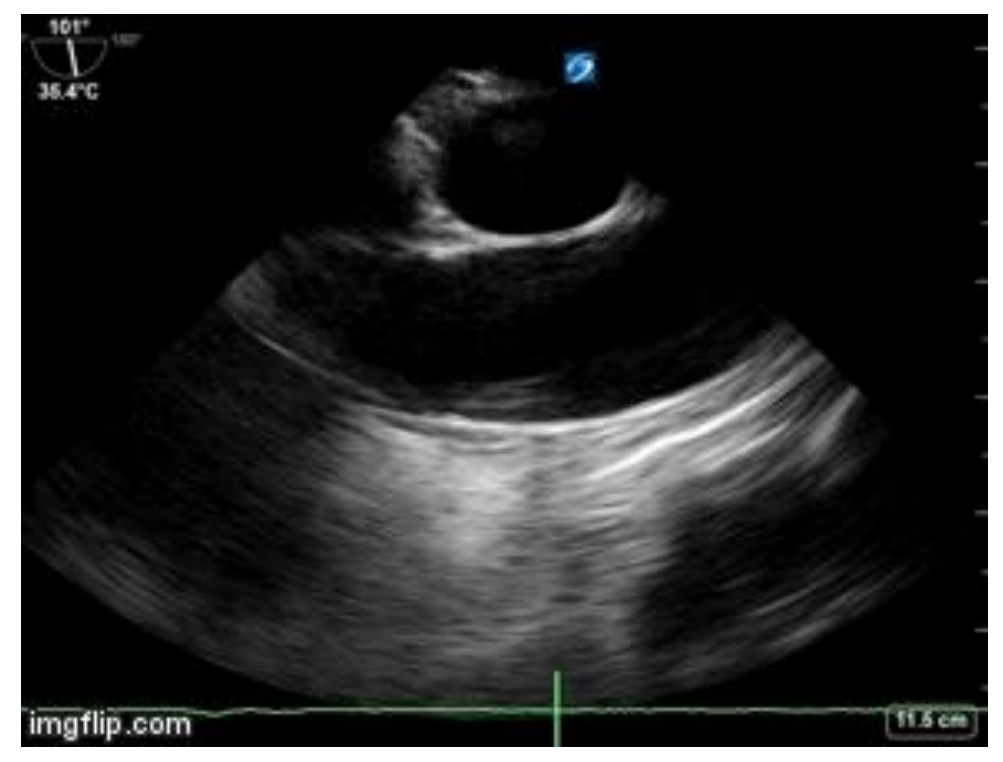

Video 3. Upper esophageal short axis view of the pulmonary artery with the ascending aorta in long axis

Based on the images presented what do you suspect is the etiology of the patient's cardiac arrest?

1. Massive Pulmonary Embolism

2. Myocardial infarction

3. Pericardial Tamponade

4. Unable to determine 


\section{Correct! \\ 4. Unable to determine}

A hyperechoic area is seen with the right main pulmonary artery (Figure 1).

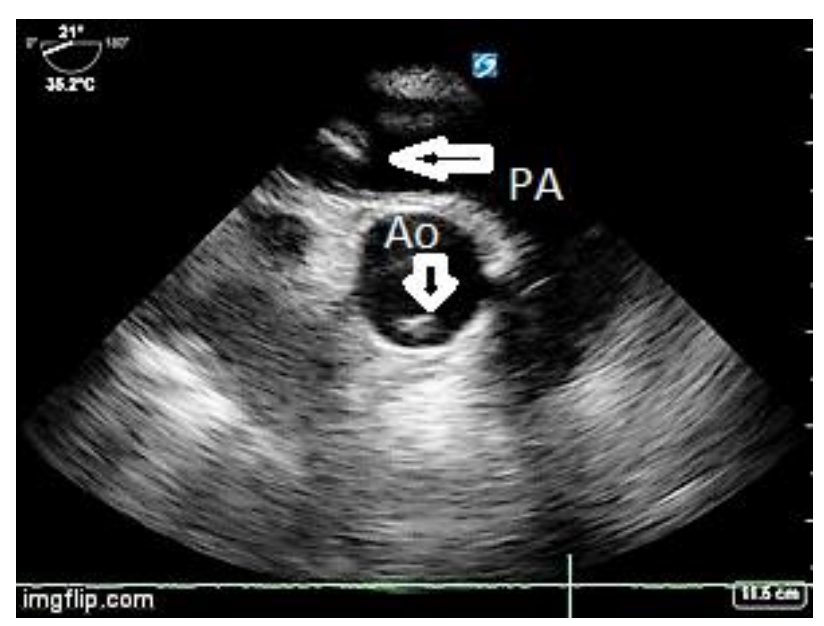

Figure 1. Upper esophageal long-axis view of the pulmonary artery. The pulmonary artery (PA) and Ascending Aorta are labeled (Ao). The left pointing arrow is at a hyperechoic area seen within the right main pulmonary artery concerning for clot. The down-pointing arrow notes an incidental reverberation artifact in the aorta.

Although this is suspicious for pulmonary embolus, the echocardiographer was concerned that this could be an artifact due to reverberation, side lobe artifact or nearfield clutter (1). Additionally, the absence of right ventricular dilatation on the 4-chamber view made the echocardiographer more suspicious that this echogenicity might not represent a true finding. More views were obtained with color Doppler in an attempt to determine if flow went around the hyperechoic area, as would be consistent with a clot (Videos 4 and 5).

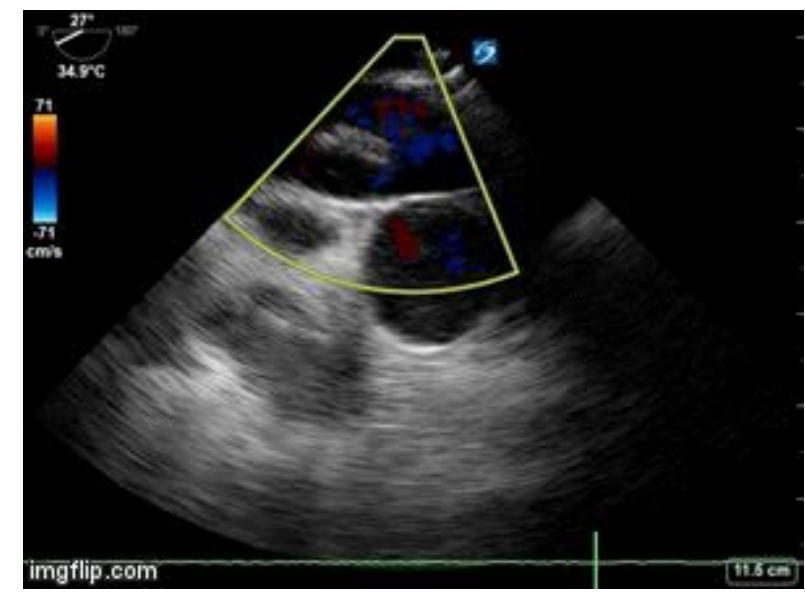

Video 4. Upper esophageal long-axis view of the pulmonary artery and short axis view of the ascending aorta with color Doppler. 


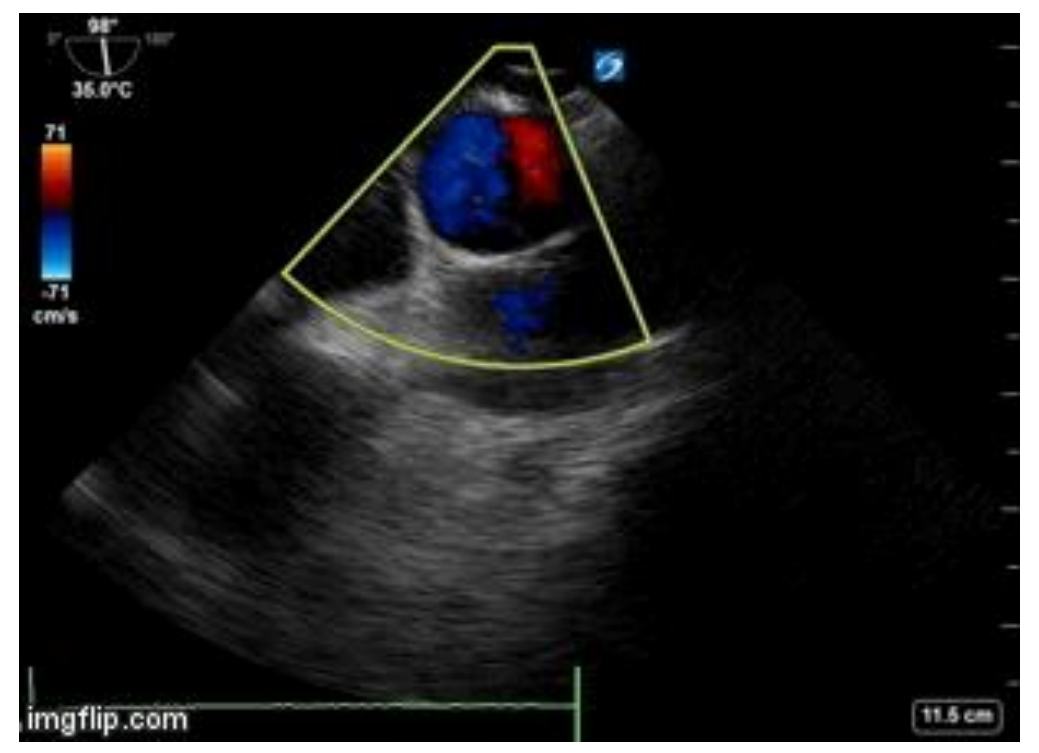

Video 5. Upper esophageal short axis view of the pulmonary artery with the ascending aorta in long axis with color Doppler.

Viewing the videos, the Doppler signal seems to go through the hyperechoic area (suggesting artifact), although this is not clearly definitive. An off-axis view was obtained that appeared to demonstrate that the hyperechoic structure went beyond the walls of the pulmonary artery (Video 6).

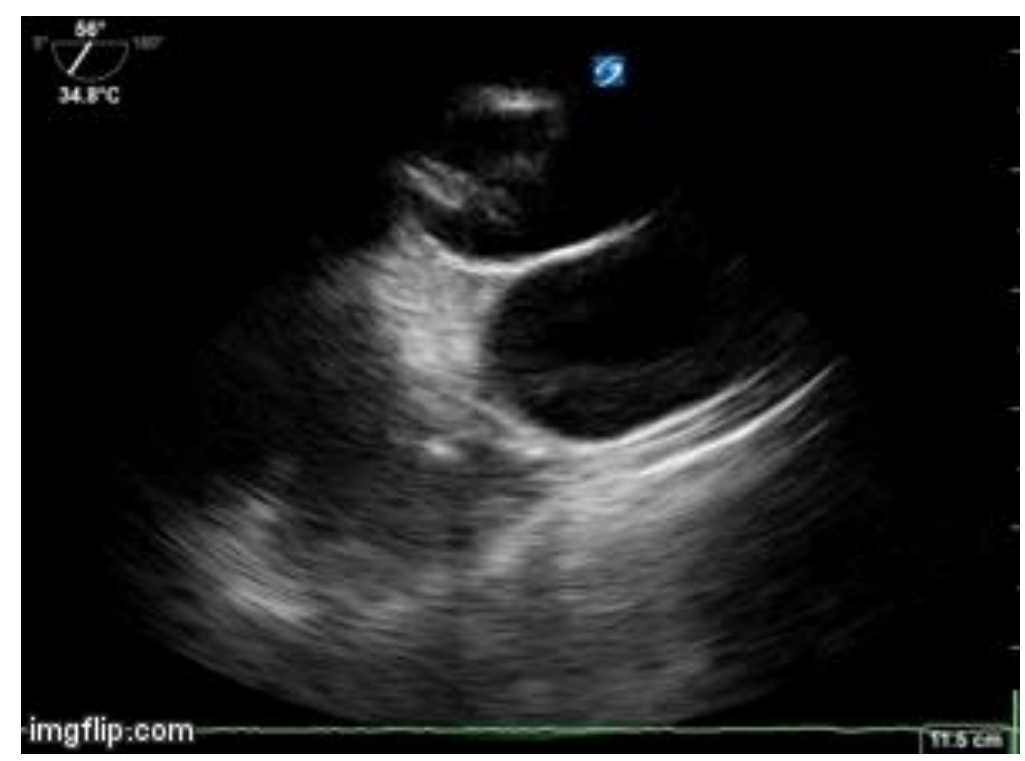

Video 6. Upper esophageal oblique view of the pulmonary artery. Artifact traversing the wall of the pulmonary artery.

Given the uncertain nature of the patient's decompensation, a computerized tomographic angiogram of the chest was performed. It demonstrated a pneumothorax and atelectasis, but no filling defects were seen in the pulmonary artery (Figure 2). 


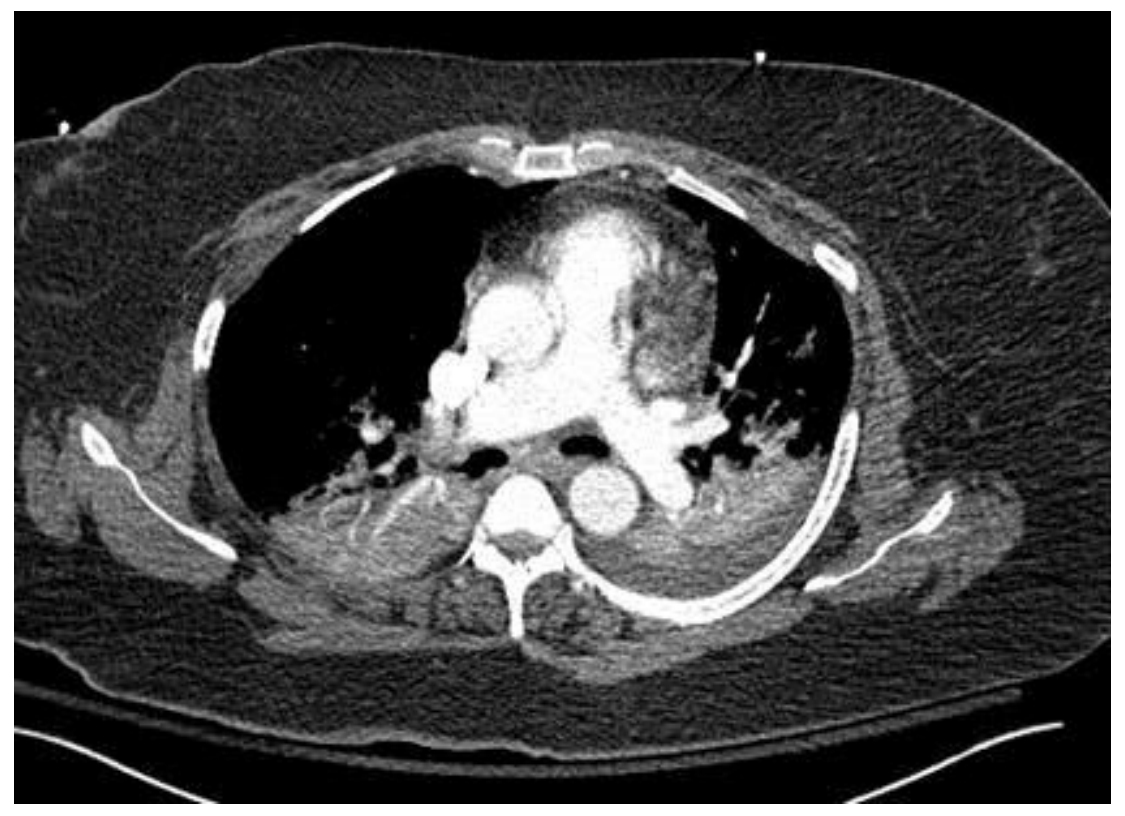

Figure 2. Representative image from CT angiogram of the main pulmonary arteries, demonstrating an absence of a filling defect, at a similar level as the TEE view was obtained.

Ultimately it was determined that the patient's cardiac arrest was due to a primary respiratory arrest caused by asthma and pneumonia. This potentially misleading artifact in TEE has been demonstrated before (2). The case emphasizes the importance of being aware of imaging artifacts when interpreting ultrasound studies.

\section{References}

1. Pamnani A, Skubas NJ. Imaging artifacts during transesophageal echocardiography. Anesth Analg. 2014;118:516-20. [CrossRef] [PubMed]

2. Liang $\mathrm{Y}$, Alvis $\mathrm{B}$, Rice $\mathrm{MJ}$, et al. A near-field clutter artifact mimicking pulmonary thrombus during transesophageal echocardiography. Anesth Analg. 2016;123:831-3. [CrossRef] [PubMed] 